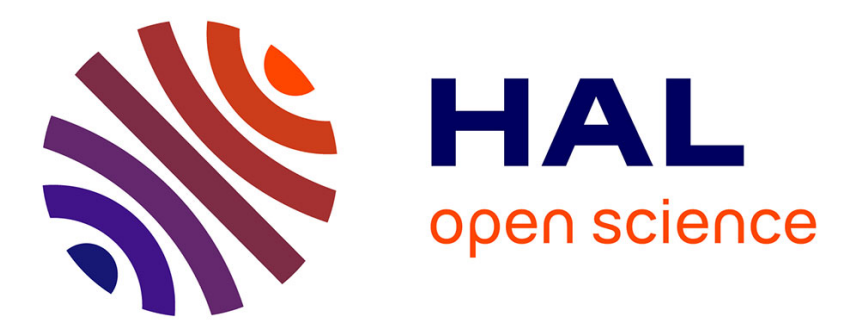

\title{
Analysis of the Retrodirective Mechanism of a Flattened Dihedral
}

\author{
Hussein Srour, R. Gillard, S. Méric, D Seetharamdoo
}

\section{To cite this version:}

Hussein Srour, R. Gillard, S. Méric, D Seetharamdoo. Analysis of the Retrodirective Mechanism of a Flattened Dihedral. IET Microwaves Antennas and Propagation, 2018, 12 (5), pp.699-705. 10.1049/iet-map.2017.0491 . hal-01724724

\section{HAL Id: hal-01724724 \\ https://hal.science/hal-01724724}

Submitted on 6 Mar 2018

HAL is a multi-disciplinary open access archive for the deposit and dissemination of scientific research documents, whether they are published or not. The documents may come from teaching and research institutions in France or abroad, or from public or private research centers.
L'archive ouverte pluridisciplinaire HAL, est destinée au dépôt et à la diffusion de documents scientifiques de niveau recherche, publiés ou non, émanant des établissements d'enseignement et de recherche français ou étrangers, des laboratoires publics ou privés. 


\begin{abstract}
In this journal, an explicit analysis of the retrodirective mechanism of a flattened dihedral using periodic arrays is proposed. Once flattened, a corner dihedral requires a phasing mechanism to restore its retrodirective behavior. This can be achieved by equipping each of the panels with passive phase-shifting cells that obey a global retrodirective phase law. The challenge is to have a trade-off between two steering mechanisms on each side such that this global phase law is achieved. In this work, an analytical study of the reflection phenomenon between the two panels is presented and sufficient information are provided to predict the retrodirective performance affected by the dihedral geometrical configuration itself. The analytical formulation of the global phase law is thus defined along with other complementary formulations to provide a better understanding of the reflection phenomenon involved. Based on these analysis and comprehension, guidelines are suggested in terms of the cell design and the dihedral geometrical configuration so that to efficiently engineer the retrodirective profile of the dihedral. A simple method utilizing these guidelines is proposed to enhance the flattened dihedral performances. And an experimental validation of this design is finally proposed at $24 \mathrm{GHz}$.
\end{abstract}




\title{
Analysis of the Retrodirective Mechanism of a Flattened Dihedral
}

\author{
H. Srour ${ }^{1,2}$, R. Gillard ${ }^{* 1}$, S. Méric ${ }^{1}$, and D. Seetharamdoo ${ }^{2}$ \\ ${ }^{1}$ Institut National des Sciences Appliquées de Rennes (INSA Rennes), \\ France \\ ${ }^{2}$ Univ. Lille Nord de France, IFSTTAR, COSYS, LEOST, FR-59650 \\ Villeneuve d'Ascq, France
}

March 6, 2018

\section{Introduction}

Backscattering enhancement is a desired feature for several civil and military applications but has unfortunately not been the subject of extensive research recently. This feature is commonly acheived by introducing a retrodirective reflector on the target such that the signal at the selected frequency is sent back in the direction of incidence $[1,2,3]$ by conjugating the phase of the wave along the receiving axis.

Several techniques have been proposed to realize analog as well as digital phase conjugation systems. For analog systems, the most common techniques are based on either passive Van Atta array [4] or on active heterodyne technique using phase conjugate mixer (PON array) [5], phase detection and Phase-Locked Loop (PLL) technique [6]. Van Atta array uses a network of transmission lines to pair arrays of distinct antennas with each other. The incoming plane wave is first received by the antennas at one end. It then propagates along the transmission lines where its initial phase is conjugated, and is finally re-radiated by the antennas at the other end of the pair. The resultant of the interference of all the re-radiated waves along the the axis of the array is a reconstructed wave traveling back in the direction of the

*raphael.gillard@insa-rennes.fr 
source. Despite being simple and reliable over a wide range of incidence angles, this topology suffers from losses in transmission lines. Modified versions of Van Atta array have been proposed with the possibility of bi-directional amplification [7], albeit, at the expense of high cost and added complexity.

Heterodyne techniques use phase conjugation mixers to reverse the incoming wave. This can be done by two main methods, either heterodyne technique using a local oscillator (LO) at twice the radio frequency (RF) [8], or heterodyne technique using intermediate frequency (IF) [9]. Phase detection and PLL techniques use more expensive and complicated systems to obtain better performance [6]. In digital systems, the signal is first downconverted using A/D converter, and is then processed by a digital signal processing system. At that stage, the carrier gets recovered and the digital signal gets demodulated, modulated and up converted [10].

Those above mentioned active retrodirective reflectors suffer from high complexity and cost. Hence, such solutions are not attractive for massmarket applications where cost and simplicity are major concerns such as for the automotive RADAR industry where backscattering is also a very desirable feature. Indeed, RADAR systems are increasingly deployed and are becoming key elements in road safety issues in a context where connected and autonomous vehicles are progressively introduced.

For automotive RADARs, a methodology based on passively redirecting waves using simple conventional metallic corner dihedral [11] can prove to be attractive due to its passive nature and the characteristic retrodirective profile it proposes for a wide range of incidence angles. However, because of its bulky structure, there is a growing interest in finding solutions to lower its profile while preserving its performances. The work presented in this paper will focus on this point.

Transformation Optics technique has been proposed [12] to reach low profile metallic corner dihedral. An adequate linear coordinate transformation is defined and realized using anisotropic homogeneous layers. However, the fabrication process is challenging. A simpler alternative is to first flatten the corner dihedral and to propose a phase compensating mechanism on each of its panels based on the reflectarray approach. In a previous work, as a proof of concept, a retrodirective flattened dihedral has been proposed at the operating frequency of $9 \mathrm{GHz}$ and at a nominal incidence angle [13]. In a later work, this design was transposed to $24 \mathrm{GHz}$, a nominal frequency allocated for short-range automotive RADARs [14]. A theoretical analysis and full-wave simulation results of the reflection mechanism between the panels was also detailed [15].

In this paper, together with an extensive analytical study on the retro- 
reflection mechanism of a flattened dihedral, experimental validations are proposed at $24 \mathrm{GHz}$. Based on the proposed analytical study, the potentials and limits on the retrodirective performance can be predicted with respect to the geometrical parameters of the configuration and the incidence angle of the incoming wave. Full-wave simulations of a nominal configuration is then proposed and validated experimentally.

The paper is organized as follows. In section 2 , the principle of operation of a flattened dihedral using reflectarray approach is briefly described. Section 3 presents the retrodirective phase law to apply on the panels of the flattened dihedral. It is derived as a function of the configuration parameters as well as the incidence angle. Section 4 distinguishes between the ideal and attainable reflection mechanisms with the latter being deployed in the design of the dihedral cells. Based on the derivation in section 3 , complementary formulations are defined in sections 5 and 6 to study the two critical elements influencing the retrodirective performance: the impact of the flattening degree and the phase law dependence on incidence angle respectively. In section 7 , the cells upon which the dihedral operates are designed and a flattened dihedral using circular cells is prototyped. Using the previous analytical derivations, a simple method utilizing elliptical cells is then proposed to enhance the reflection mechanism and hence the RCS profile of the dihedral. In section 8 , full wave simulations are performed on a design for an operation at $24 \mathrm{GHz}$, in which the obtained results are accompanied by relevant interpretations to validate the theoretical findings. Finally, an experimental validation is given in section 9 .

\section{Principle of Operation}

In the context of this work, the dihedral corner is significantly flattened in such a way it totally loses its desired retrodirective characteristic. To restore this property, a steering mechanism is built on its panels $[13,15]$.

This mechanism consists of two arrays of well tuned phase-shifting cells. The oppositely placed arrays follow a predefined global phase law materialized by an incremental phase shift $(\gamma)$ as shown in Fig. 1. The phase shift is defined in function of the structure parameters and the incidence angle of the incoming wave. 


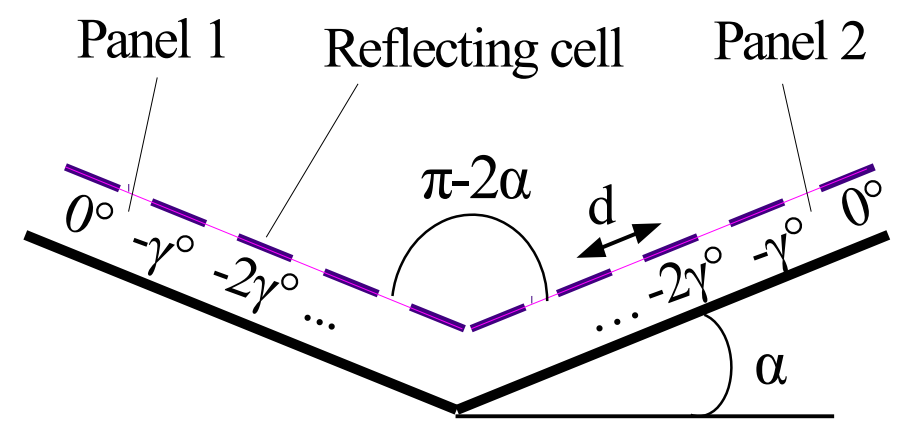

Figure 1: Side view of the configuration of the flattened dihedral

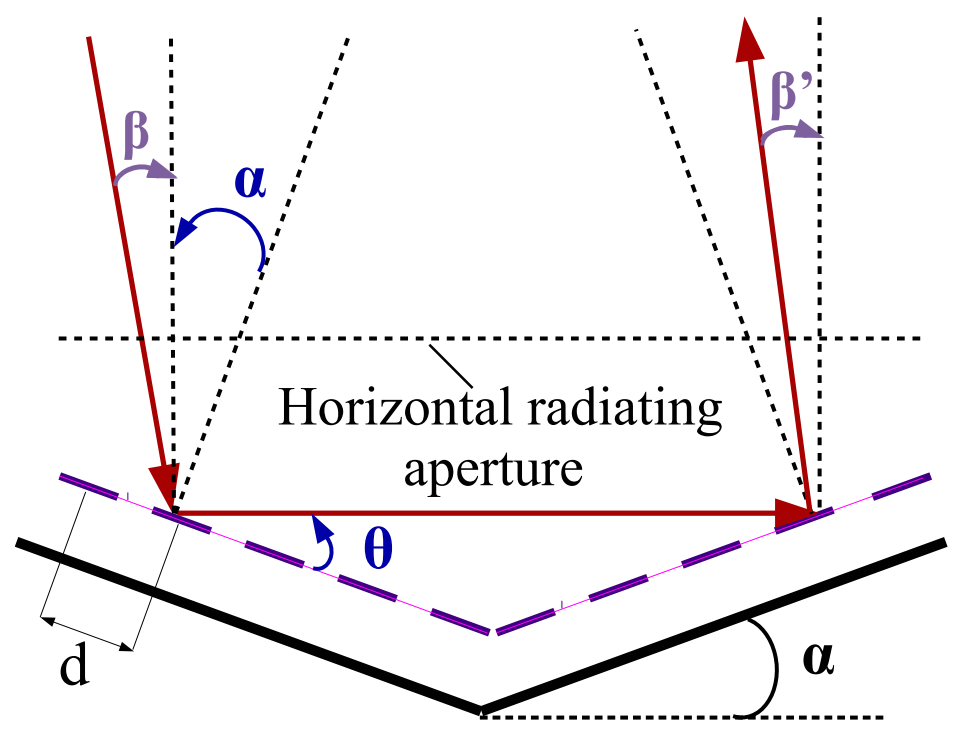

Figure 2: Dihedral sketch with the important notations: the flattening degree $\alpha$, the inter-element spacing $d$, the incidence angle $\beta$ of the incoming wave, the angle $\theta$ upon which the beam leaves the first panel and the angle $\beta^{\prime}$ corresponding to the beam leaving the second panel

\section{Phase Law Derivation}

In this part, a detailed analytical derivation of the phase law is presented. Fig. 2 shows a sketch of the dihedral configuration with all the needed angle notations for this derivation. 


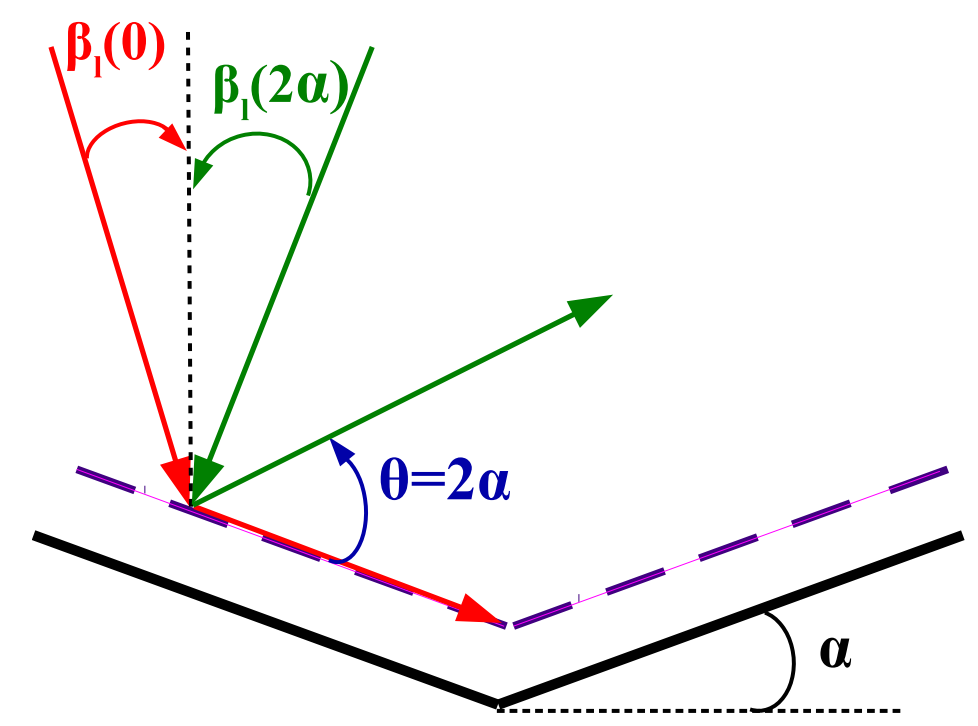

Figure 3: Illustration of the limiting range using $\beta_{l}$ and $\theta$ angles

In a previous work [13], the phase law has been derived independently from the incidence angle and more precisely only for the nominal case where the incident wave arrives normally to the horizontal radiating aperture $(\beta=0$ in Fig. 2).

The phase law, materialized in the form of phase shift, is derived in function of the incidence angle $\beta$ of the incoming wave, the flattening degree $\alpha$, inter-element spacing $d$, and the wave number $k_{0} \cdot \gamma^{\beta}$, the phase gradient between two consecutive cells, can be formulated using simple trigonometric formulations that describe the reflection from one panel to the other.

It is important to note that two reflection configurations can be distinguished. When the beam first hits panel 1 and then leaves the dihedral from panel 2, the "forward" reflection is being referred to. When the opposite happens, then the "reverse" reflection is being referred to. Due to the geometrical symmetry, only the forward reflection will be discussed and similar conclusions can be obtained for the reverse case.

The beam intercepts the panel 1 with an angle $\beta$ with respect to the vertical and leaves with angle $\theta$. The combination of the imposed phase shift, $\gamma_{\beta}$, in addition to the delay resulting from the oblique incidence angle $\alpha-\beta$ yields the following equation:

$$
\gamma_{\beta}+k_{0} d \sin (\alpha-\beta)=k_{0} d \cos (\theta)
$$


Then, the beam intercepts panel 2 and leaves by angle $\beta^{\prime}$ :

$$
-\gamma_{\beta}+k_{0} d \cos (2 \alpha-\theta)=k_{0} d \cos \left(\pi / 2-\alpha-\beta^{\prime}\right)
$$

The condition so that the beam is redirected back in the direction of incidence is:

$$
\beta^{\prime}=\beta
$$

By substituting equation 3 in 2, a system of two equations and two unknowns is obtained, which can be solved using simple trigonometric relations. Finally, the phase shift to be applied becomes:

$$
\gamma_{\beta}=k_{0} d\left(\cos \left(\alpha+\arcsin \left[\frac{\sin (\beta)}{\tan (\alpha)}\right]\right)-\sin (\alpha-\beta)\right)
$$

Specifically, for the nominal case where $\beta=0$, the phase law becomes:

$$
\gamma_{0}=k_{0} d(\cos (\alpha)-\sin (\alpha))
$$

This is the same formulation obtained in [13].

With the phase law being formulated as a function of the incidence angle $\beta$, a study will be conducted later on to assess the impact of this sensitivity on the performance of the reflector.

\section{Ideal and Attainable Reflections}

Before going deeper in this study, there is a necessity to distinguish between the attainable and ideal reflections. We assume that the reflection is ideal when the cells can be reconfigured to match the phase shift (4) required for any incident angle. This means that the reflector should be able to sense the direction from which the beam is coming and reconfigure its cells to adjust to the phase law for this specific angle. This would require a huge electronic system which is not a feasible solution. However, this ideal situation is of notable importance because it can help us assess the impact of the flattening degree while totally neglecting any phase law inaccuracies. The attainable case is the one where cells are tuned to realize the progressive phase shift corresponding to a specific incidence angle. This is exactly what we are trying to emulate. A study dedicated for this case might give us a good idea about how our fabricated prototype might perform. 


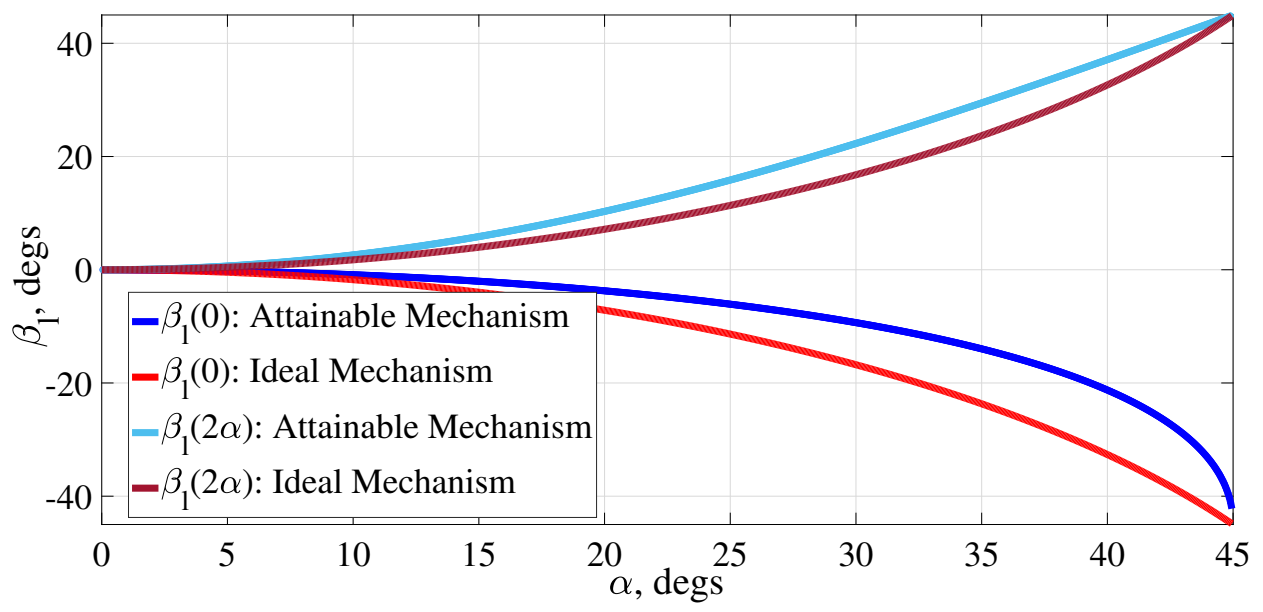

Figure 4: $-\beta_{l}$ versus $\alpha$ for the ideal and attainable cases

\section{$5 \quad$ Study on the Flattening Degree}

In this section, the effect of the flattening degree on the retrodirective performance of the flattened dihedral is studied. To do so, a new parameter $\beta_{l}$ is defined as a function of the flattening degree $\alpha$ for both the ideal and attainable cases. The angle $\beta_{l}$ describes the limiting range in which the reflected beam from the first panel is intercepted by the second panel. The value of $\beta_{l}(0)$ corresponds to the limit where $\theta=0^{\circ}$ and $\beta_{l}(2 \alpha)$ corresponds to the limit where $\theta=2 \alpha$. An illustration is provided in Fig. 3. Thus, the performance is expected to degrade for incidence angles outside this range.

$\theta$ for the ideal case can be deduced from equations (1) and (4) as following:

$$
\theta=\alpha+\arcsin \left(\frac{\sin (\beta)}{\tan (\alpha)}\right)
$$

In this case, it is found that $\beta_{l}(2 \alpha)=-\beta_{l}(0)$ with the latter being obtained by forcing $\theta$ to $0^{\circ}$ :

$$
\beta_{l}(0)=-\arcsin (\sin (\alpha) / \tan (\alpha))
$$

For the attainable case, $\theta$ can be obtained from equation (1) by locking the phase shift to a nominal incidence angle $\beta_{0}$. $\theta$ can be hence written in this form:

$$
\theta=\arccos \left(\frac{\gamma_{\beta_{0}}}{k_{0} d}+\sin (\alpha-\beta)\right)
$$




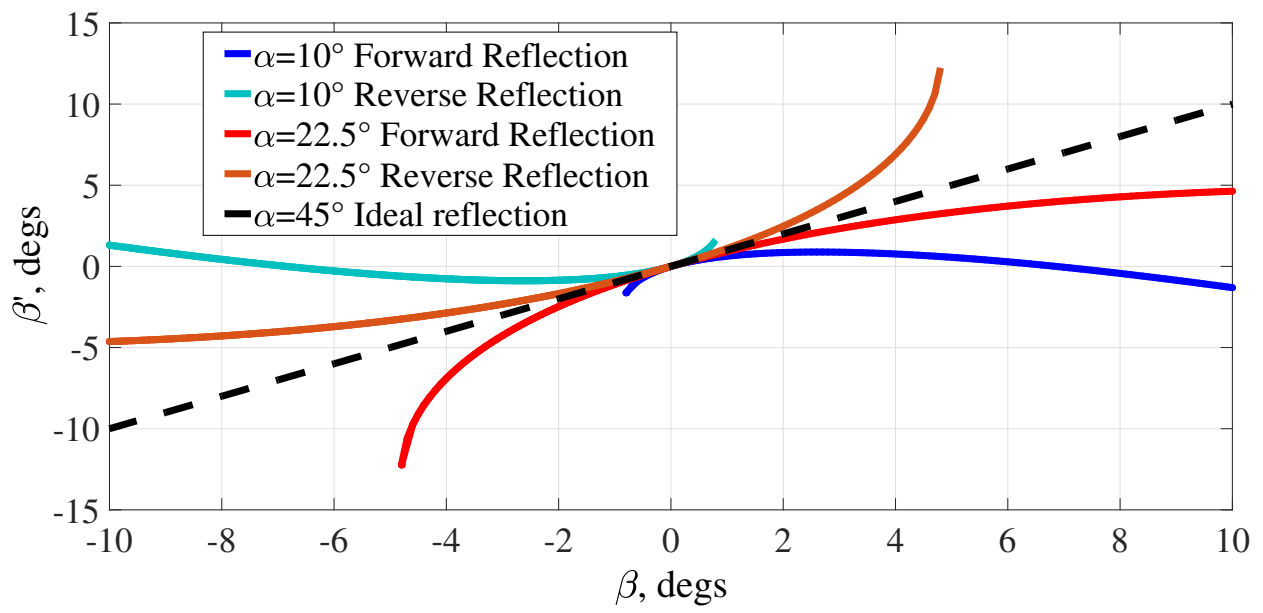

Figure 5: $\beta^{\prime}$ versus $\beta$ for the forward and reverse functions

Here, $\beta_{l}(0)$ and $\beta_{l}(2 \alpha)$ have different values and can be obtained from $\theta$ as following:

$$
\begin{gathered}
\beta_{l}(0)=\alpha-\arcsin \left(1-\frac{\gamma_{\beta_{0}}}{k_{0} d}\right) \\
\beta_{l}(2 \alpha)=\alpha-\arcsin \left(\cos (2 \alpha)-\frac{\gamma_{\beta_{0}}}{k_{0} d}\right)
\end{gathered}
$$

In Fig. $4, \beta_{l}(0)$ and $\beta_{l}(2 \alpha)$ are plotted in function of $\alpha$ for the ideal and the attainable cases. For the latter, the phase shift is obtained for the nominal case $\left(\beta=0^{\circ}\right)$ and the inter-element spacing over wavelength ratio $\mathrm{d} / \lambda$ is fixed to $1 / 3$. As expected, $\beta_{l}(0)=-\beta_{l}(90)=-45^{\circ}$ is obtained for $\alpha=45^{\circ}$ (classical dihedral). Both $\beta_{l}(0)$ and $\beta_{l}(2 \alpha)$ decrease whenever the dihedral is flattened, in other words, as $\alpha$ decreases. It is interesting to find out that even for the ideal case the limiting range decreases quite rapidly with $\alpha$. The curves also show that the lower limit $\beta_{l}(0)$ for the ideal case is greater than that of the attainable case. The difference increases up to $20^{\circ}$ degrees for certain flattening degrees. However, the upper limit $\beta_{l}(2 \alpha)$, is greater for that of the attainable case. Also, the absolute value of the upper limit $\beta_{l}(2 \alpha)$ of the attainable case is always greater than that of the absolute value of the lower limit $\beta_{l}(0)$ of the ideal case. Further discussion will follow in section 8. 


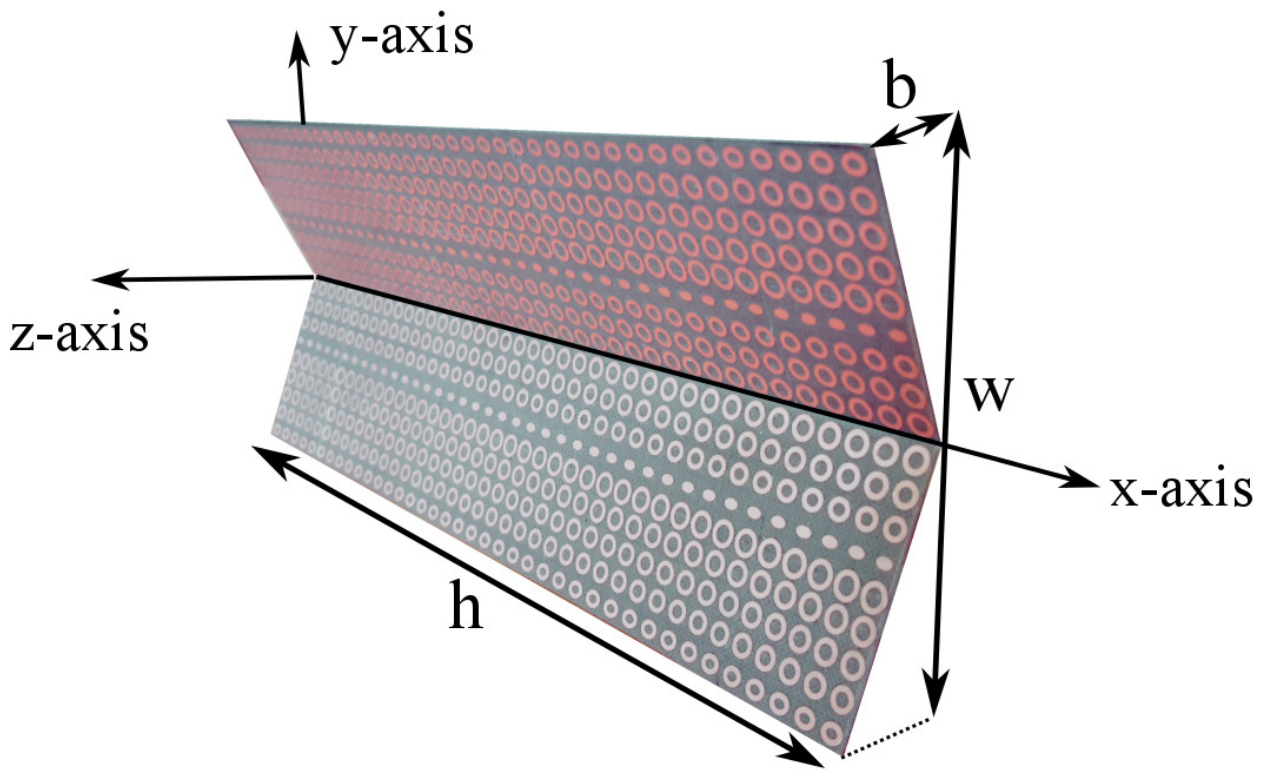

Figure 6: 3D view of the proposed flattened dihedral using elliptical cells

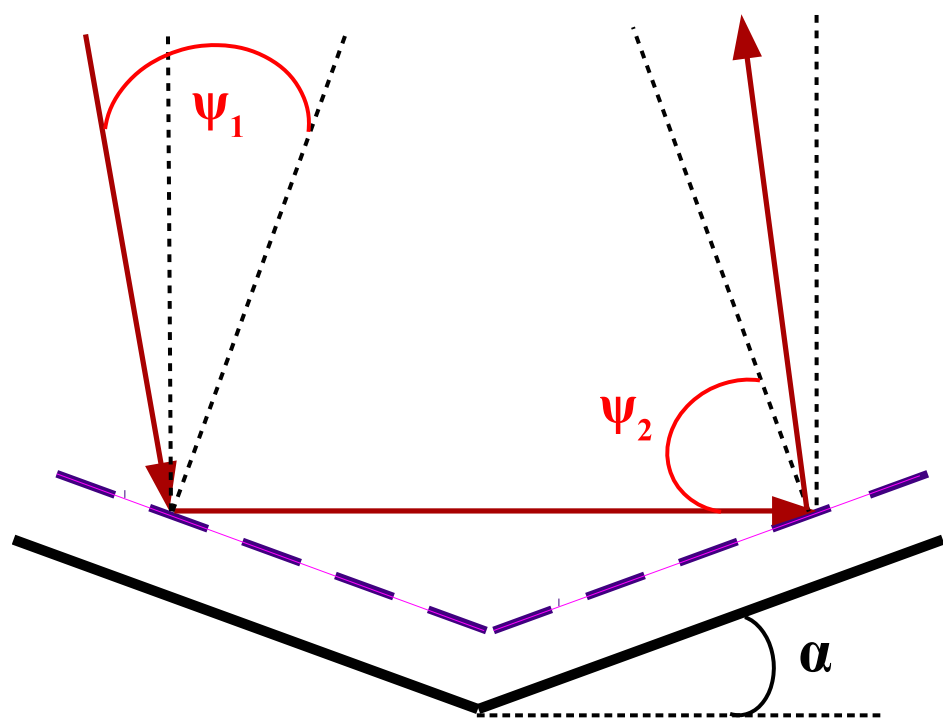

Figure 7: Definition of the angles of incidence $\psi_{1}$ and $\psi_{2}$ on each of the panels 1 and 2 respectively 


\section{Study on the Phase Law Dependence on Inci- dence Angle}

In this part, the impact of phase law inaccuracy over the range of incidence angles is assessed. As illustrated in Fig. 2, a new metric $\beta^{\prime}$ is defined as the angle which describes the direction of maximum radiation of the reflected beam with respect to the normal of horizontal aperture.

In the ideal case, $\beta^{\prime}$ is simply equal to $\beta$, while for the attainable case, $\beta^{\prime}$ can be deduced from equation 2 upon fixing a predefined nominal phase shift. $\beta^{\prime}$ can be written for the forward and reverse reflections respectively as:

$$
\begin{aligned}
\beta^{\prime}=-90^{\circ}-\alpha-\arccos ( & \frac{\gamma_{\beta_{0}}}{k_{0} d} \\
& \left.+\cos \left(\alpha-\arccos \left(\frac{\gamma_{\beta_{0}}}{k_{0} d}+\sin (\alpha-\beta)\right)\right)\right)
\end{aligned}
$$

and

$$
\begin{aligned}
\beta^{\prime}=-90^{\circ}+\alpha+\arccos ( & -\frac{\gamma_{\beta_{0}}}{k_{0} d} \\
& \left.+\cos \left(\alpha-\arccos \left(\frac{\gamma_{\beta_{0}}}{k_{0} d}+\sin (\alpha+\beta)\right)\right)\right)
\end{aligned}
$$

In Fig. 5, the ideal and the attainable $\beta^{\prime}$ are plotted assuming that $\alpha=22.5^{\circ}$ and inter-element spacing $d / \lambda=1 / 3$. As expected, the reflector behaves perfectly for incidence angle $\beta=0^{\circ}$. The attainable $\beta^{\prime}$ still manages a deviation less than $5^{\circ}$ for incidence angles $-5^{\circ}<\beta<5^{\circ}$. However, outside this range, attainable $\beta^{\prime}$ deviates drastically for the incidence angles where it is still defined. This leads to conclude that there are three regions being operated by the reflector:

1. The region where the forward and the reverse reflection configurations are operating simultaneously: here we expect maximum performance from the reflector with a peak at the incidence angle corresponding to the nominal case $\left(\beta=0^{\circ}\right.$ here).

2. The region where only the forward configuration is operating: the performance degrades as $\beta$ positively increases.

3. The region where only the reverse configuration is operating: the performance degrades as $\beta$ negatively increases. 
Those findings can be used to predict the RCS profile of the designed flattened dihedral, thus, creating guidelines to follow regarding the design of this reflector.

\section{Application using Annular Reflectarray Cells}

In this section, a design of a flattened dihedral using annular cells is proposed. Operating at $24 \mathrm{GHz}$, this dual-polarized reflector can be of great interest for collision avoidance systems. As shown in Fig. 6, the dihedral array is composed of 9 annular cells of inter-element spacing equal to $d=\lambda_{0} / 3$. Those 9 cells are repeated 39 times in the x-axis direction so that to obtain an aperture effective area equivalent to that of a classical dihedral with a theoretical maximum RCS of $10 \mathrm{dBsm}$.

Following the most-likely scenario, the dihedral is designed to operate for the nominal case defined earlier. The corresponding incremental phase shift $\gamma_{0}$ is obtained from equation 5 . Considering $\lambda_{0}=12.5 \mathrm{~mm}, k_{0}=2 \pi / \lambda_{0}$, $d=\lambda_{0} / 3$ and $\alpha=22.5^{\circ}$, the phase shift becomes:

$$
\gamma_{0}=k_{0} d(\cos (\alpha)-\sin (\alpha)) \approx 64.95^{\circ}
$$

Simple annular patches possess phase response that provides phase control over a sufficiently wide range to achieve the required phase law. The corresponding phase shift at a certain unit cell is obtained by tuning the patch dimension to a specific radius while keeping its width fixed.

In a classical design, the cells dimensions are obtained assuming a plane wave at normal incidence to the panel. However, this setup might not be appropriate in the case of a complex structure composed of two attached panels. In such a topology, the beam hits the two panels with two very different incidence angles $\psi_{1}$ and $\psi_{2}$ as shown in Fig. 7. This introduces inescapable errors due to the dependency of the phase response to incidence angle.

To illustrate, figures 8 and 9 plot the phase response versus cell radius for different incident angles in Transverse Electric ( $\mathrm{E}$ field along $\mathrm{x}$-axis) and Transverse Magnetic ( $\mathrm{H}$ field along $\mathrm{x}$-axis) configurations respectively. The cell consists of an annular patch of fixed width $=0.8 \mathrm{~mm}$ supported by a Rogers substrate $0.787 \mathrm{~mm}$ thick with $\epsilon_{r}=2.2$ and loss tangent $\tan \delta=0.02$. This cell response has been analyzed using the infinite array model ([16],[17]) integrated in Ansys HFSS software.

The maximum simulated loss in the cell is $0.07 \mathrm{~dB}$ whatever the patch radius and the polarization of the wave. This is consistent with the fact that 


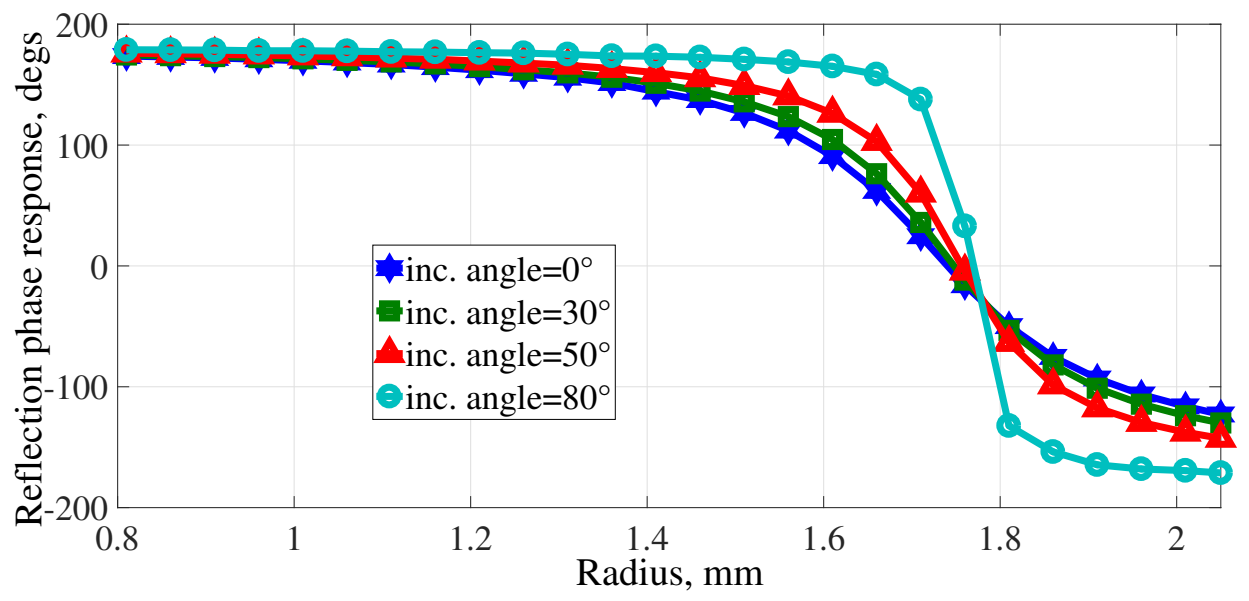

Figure 8: The reflection phase response versus radius for different incidence angles in the TE polarization

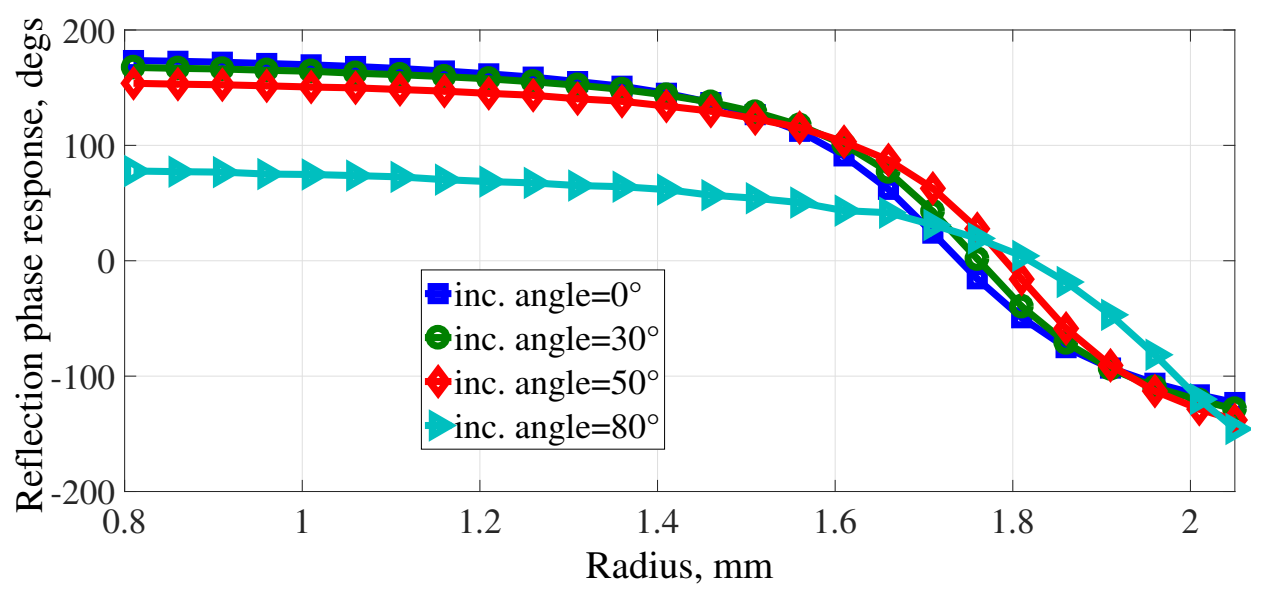

Figure 9: The reflection phase response versus radius for different incidence angles in the TM polarization

the cell is backed by a ground plane and uses low-loss materials. From the phase curves, it can be seen that the appropriate radius is highly related to the incident angle. The main concern here is that the incident beams on each of the two panels arrive in two different incident angles: one directly coming from the source and the other coming from the adjacent panel (after a first reflection). This was not taken into account in the previous work [13]. 
Table 1: Cells dimensions $(\mathrm{mm})$ based on the classical and the proposed designs

\begin{tabular}{|c|c|c|c|}
\hline Phase-shift(deg.) & Normal incidence & $45^{\circ} \mathrm{TE}$ & $45^{\circ} \mathrm{TM}$ \\
\hline & circular & elliptical-major & elliptical-minor \\
\hline 129.9 & 1.5 & 1.6 & 1.39 \\
\hline 64.95 & 1.66 & 1.7 & 1.7 \\
\hline 0 & 1.75 & 1.76 & 1.78 \\
\hline-64.95 & 1.84 & 1.8 & 1.86 \\
\hline-129.9 & 2.05 & 1.91 & 2.0 \\
\hline 165.15 & 1.01 & 1.22 & 0.81 \\
\hline 100.2 & 1.59 & 1.66 & 1.6 \\
\hline 35.25 & 1.7 & 1.73 & 1.74 \\
\hline-29.7 & 1.78 & 1.78 & 1.81 \\
\hline
\end{tabular}

Figure 10: Caption of the circular and elliptical cells of the retrodirective flattened dihedral

In fact, the cells were tuned assuming normal incidence on both panels. This is referred as the classical simulation setup. A new setup has been proposed in [15] where the design is achieved for an average incident angle that is a trade-off between the incidence angle on panel $1, \psi_{1}=\alpha=22.5^{\circ}$ (nominal case), and the corresponding incidence angle on panel $2, \psi_{2}$.

It can be simply derived that this average angle is $45^{\circ}$.

To demonstrate the difference between the phase profiles of the classical and the new setups, Fig. 11 plots the absolute value of the phase deviation when changing the incidence angle from $0^{\circ}$ to $45^{\circ}$ for TE and TM configurations. This plot shows that the phase deviation can reach up to $41^{\circ}$ and $43^{\circ}$ in TE and TM configurations respectively.

Since the incidence is non-normal, the design should be different for TE (E field along $\mathrm{x}$-axis) and TM ( $\mathrm{H}$ field along $\mathrm{x}$-axis) polarizations leading 


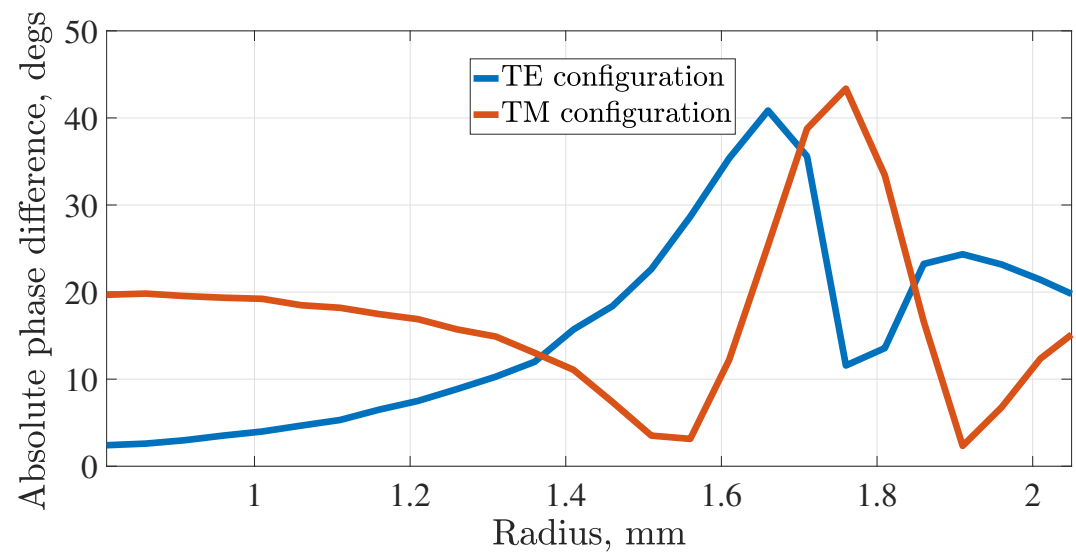

Figure 11: Absolute value of phase deviation between the new and classical phase profiles for the TE and TM configurations

to non-identical cells along both axes. The dimensions of those elliptical cells alongside the circular ones corresponding to the classical setup are shown in Tab. 1 for each of the required phase shifts. The cell dimension corresponding to phase shift $165.15^{\circ}$ could not be covered by the obtained phase range and hence is set to the closest value which is $0.81 \mathrm{~mm}$. The chosen circular and elliptical cells are captured in Fig. 10.

\section{Simulation Results and Discussion}

In this section, the results of full wave simulations for the designs proposed in the previous part are presented and discussed. Two major points are being looked after here:

1. Validation of the theoretical analysis done in sections 5 and 6 .

2. Validation of the method proposed in the previous section.

For a complete comparison, a classical dihedral corner, a bare flattened dihedral and flattened dihedrals with circular and elliptical cells have been simulated using commercial software HFSS. The full description is listed in Tab. 2. Each of the above targets has been immersed in a radiating box and excited by an incident wave ranging from $-20^{\circ}$ till $20^{\circ}$ with respect to the z-axis in the (YOZ) plane. The radiating box is surrounded by perfectly matched layers to absorb the scattered wave. 
Table 2: Geometrical dimensions of reference corner dihedral and flattened dihedral

\begin{tabular}{cccc}
\hline Structure & $\mathrm{b}, \mathrm{mm}$ & $\mathrm{h}, \mathrm{mm}$ & $\mathrm{w}, \mathrm{mm}$ \\
\hline Classical dihedral & 34.65 & 162.5 & 69.29 \\
Flattened dihedral & 14.35 & 162.5 & 69.29 \\
\hline
\end{tabular}

Fig. 12 shows the monostatic RCS level of the above-mentioned structures for the TE configuration (E field along x-axis). First to notice is the massive enhancement the phasing mechanism introduces to a bare flattened dihedral specifically at normal incidence with an increase of more than 10 $\mathrm{dB}$. Comparing the RCS profile of the retrodirective flattened dihedral with that of a conventional dihedral corner shows a drop in the RCS level at normal incidence corresponding to the nominal case. This can be explained by two main factors. Firstly, the phase law that is applied on the two panels is only an approximation of the ideal phase law that should be applied. Indeed, it is a discretized version of the desired phase law (due to the limited number of cells). Moreover, the reflected phase produced by each cell is derived using infinite array model, which may be responsible for an additional slight deviation. Also, as explained before, this derivation also assumes an average incident angle contributing to further inaccuracies in the reflection process. Secondly, it is well-known that meta-mirrors suffer from the presence of lobes in the specular and parasitic directions leading to energy loss in the main beam [18]. The structure here is even more complex since it can be seen as the combination of 2 such meta-mirrors.

The analytical work done in sections 5 and 6 helps interpreting the obtained RCS profiles. First, to understand why the flat RCS profile suddenly deteriorates at a certain angle, the answers can be found in the analysis done on the limiting range in section 5. In Fig.4, the limiting range in the forward configuration of the attainable case is plotted. For this case where $\alpha=22.5^{\circ}$, it can be found that $\beta_{l}(0) \approx-5^{\circ}$ and $\beta_{l}(45) \approx 13^{\circ}$. Similarly, for the reverse configuration, it can be deduced that $\beta_{l}(0) \approx-13^{\circ}$ and $\beta_{l}(45) \approx 5^{\circ}$. This means that at least one of the two forward and reverse configurations is operating for $-13^{\circ}<\beta<13^{\circ}$. From the simulation results, it can be seen that the limiting range is nearly $-11^{\circ}<\beta<11^{\circ}$ which is in good agreement with the theoretical predictions.

On the other hand, the three regions discussed in section 6 can be clearly visualized in Fig. 12. Again, the analytical formulations for the attainable case predict maximum performance intensity-wise for incidence angles $-5^{\circ}<\beta<5^{\circ}$ for the first region. For regions two $\left(-11^{\circ}<\beta<-5^{\circ}\right)$ and 


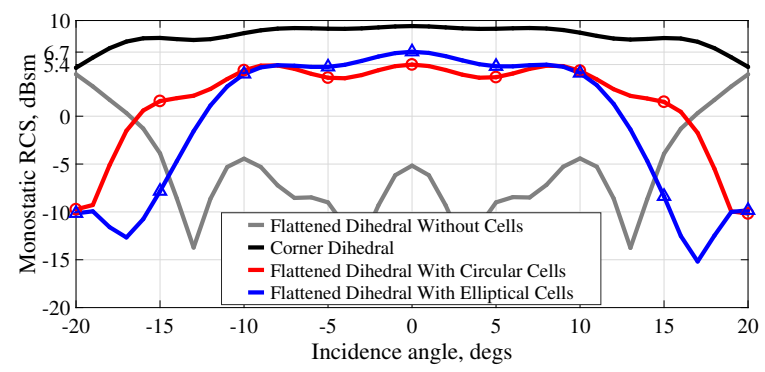

Figure 12: RCS simulations of bare, corner and retrodirective flattened dihedrals for TE case

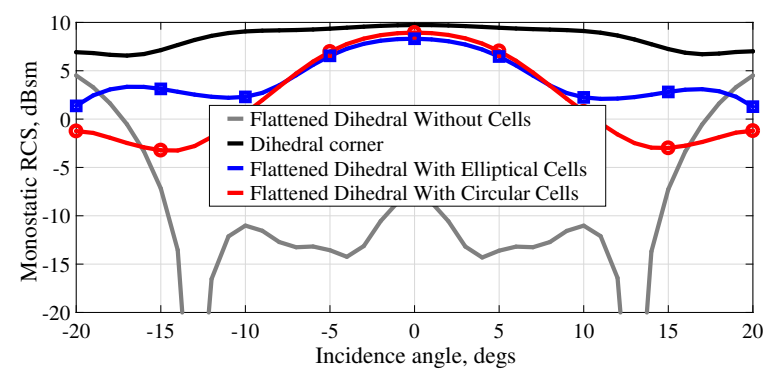

Figure 13: RCS simulations of bare, corner and retrodirective flattened dihedrals for TM case

three $\left(5^{\circ}<\beta<11^{\circ}\right)$, either forward or reverse reflection function is active, and acceptable RCS is obtained. Fig. 13 shows the dihedrals $\Delta$ RCS profiles obtained for TM configuration ( $\mathrm{H}$ field along $\mathrm{x}$-axis). Despite that the interpretations mentioned above are not so evident in the results, the RCS profile sustains high values for incidence angles around the nominal angle as compared to a dihedral corner.

Overall, the full wave simulations do validate the predictions obtained using the analytical formulations. Those findings can be seen as guidelines designers can follow in order to engineer the desired RCS using the flattened dihedral reflector.

Regarding the average-angle setup, for TE case, 1.3 dB RCS enhancement has been obtained at the nominal incidence using elliptical cells. For TM case, the RCS level is almost the same for both configurations at nominal incidence. However, the angular range is improved for the design using elliptical cells. Those results demonstrate the fact that the new proposed setup enhances the phasing mechanism and thus improves the retrodirective 


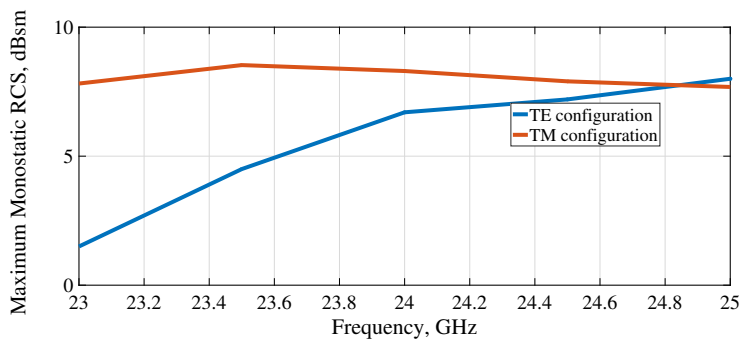

Figure 14: RCS values at $\beta=0^{\circ}$ versus frequency for TE and TM configurations

reflection process from one panel to the other and back to the source.

The flattened dihedral is now simulated for frequencies ranging between 23 to $25 \mathrm{GHz}$. Fig. 14 plots the maximum RCS value as a function of the frequency range for TE and TM configurations. The plot shows that the maximum RCS is quite stable over the whole range of frequency in TM configuration. By stable it is meant that the maximum RCS does not drop lower than $1 \mathrm{dBsm}$ from the maximum value obtained over the frequency range. In TE configuration, the plot is more stable for higher frequencies ranging between 24 and $25 \mathrm{GHz}$ and shows significant deterioration for lower frequencies.

\section{Experimental Validation}

In order to validate the simulation results, a prototype has been fabricated using laser technology to engrave the cells on the substrate. Each panel is fixed on a bare metallic dihedral structure. The measurements were performed in the IETR facility CACENDRA (ChAracterization in CENtimeter band of RAdiating devices) using two horn antennas and a network analyzer. The antennas are fixed in the farfield region of the target, which is placed on a rotating platform. This permits to measure the monostatic RCS for the whole range of incidence angle. Time gating method is incorporated in the measurements procedure. First, it transforms the frequency domain response to a time domain one. Then, by imposing a time gate, the multipath interferences can be suppressed. The resultant time domain signal is re-transformed into frequency domain and processed. Fig. 15 shows the comparison between the simulation and measurement results for the flattened dihedral using elliptical cells for TE and TM cases. The measurement results are in very good agreement with those of the simulation. In gen- 
eral, the measurement results validate the concept behind using a phasing mechanism based on phasing cells. And specifically, they show that the simple method of using a compromise setup has improved its retrodirective performance.

\section{Conclusion}

In this paper, a detailed analysis has been offered on the retrodirective mechanism of a flattened dihedral. A global phase law has been derived in function of incidence angle and geometry parameters. And a sensitivity analysis has been conducted to determine from one side the impact geometrically flattening the dihedral has on the performance, and from other side, the consequence of using inaccurate phase law on the operating range of incidence angles. The elements of this study have provided sufficient information to predict the theoretical RCS profile and thus served forming clear guidelines regarding the design of the geometrical aspects of the dihedral and the operating integrated cells. Following this study, a method to enhance the reflection mechanism between the two panels and consequently the RCS profile has been proposed followed up with an example of a flattened dihedral using annular cells. By interpreting the results of the full wave simulations, it could be shown that the theoretical analysis can be successfully used to predict the performance of such a configuration. Also, it can be shown that using a method based on average-angle setup, the RCS profile can be enhanced. Finally, an experimental validation has been given at $24 \mathrm{GHz}$. The measurements performed have shown good agreement with the simulation results and thus validate the methodology behind this work.

\section{Acknowledgments}

The authors would like to express their gratitude to ANR (Agence Nationale de la Recherche) for funding this study through the CYCLOPE (CYCLOProtection Electronique) project.

\section{References}

[1] Miyamoto R. Y. , Itoh T.: 'Retrodirective arrays for wireless communications', IEEE Microwave Magazine, 2002, 3, pp. 71-79 


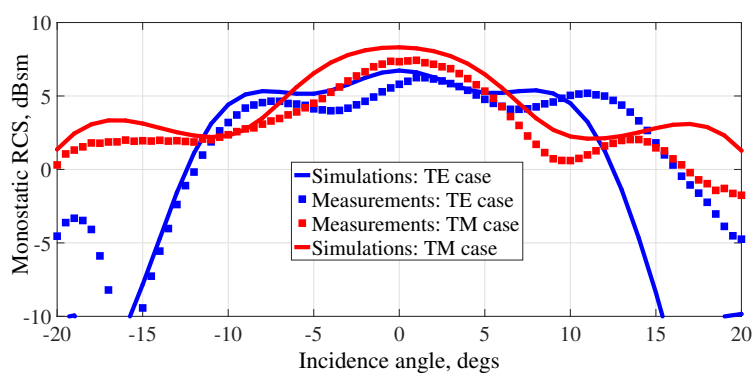

Figure 15: RCS comparisons between simulations and measurements for retrodirective flattened dihedrals using elliptical cells for TE and TM polarizations

[2] Goshi D. S. , Leong K. M. K. H., Itoh T.: 'Recent advances in retrodirective system technology', 2006 IEEE Radio and Wireless Symposium, 2006, pp. 459-462

[3] Zamora A., Iwami R. T., Chun T. F., Shiroma W. A.: 'An overview of recent advances in retrodirective antenna arrays', 2010 IEEE International Conference on Wireless Information Technology and Systems, 2010, pp. 1-4

[4] Sharp E., Diab M.: 'Van Atta reflector array', IRE Transactions on Antennas and Propagation, 1960, 8, pp. 436-438

[5] Pon C.: 'Retrodirective array using the heterodyne technique', IEEE Transactions on Antennas and Propagation, 1964, 12, pp. 176-180 in , vol. 53, no. 2, pp. 730-738, Feb. 2005.

[6] Fusco V., Chee Binn Soo, Buchanan N.: 'Analysis and characterization of PLL-based retrodirective array', IEEE Transactions on Microwave Theory and Techniques, 2005, 53, pp. 730-738

[7] Chung S. J., Chen S. M., Lee Y. C.: 'A novel bi-directional amplifier with applications in active Van Atta retrodirective arrays', IEEE Transactions on Microwave Theory and Techniques, 2003, 51, pp. 542-547

[8] Luxey C., Laheurte J. M.: 'A retrodirective transponder with polarization duplexing for dedicated short-range communications', IEEE Transactions on Microwave Theory and Techniques, 1999, 47, pp. 1910-1915

[9] Leong K. M. K. H., Wang Y., Itoh T.: 'A full duplex capable retrodirective array system for high-speed beam tracking and pointing applica- 
tions', IEEE Transactions on Microwave Theory and Techniques, 2004, 52, pp. 1479-1489

[10] DiDomenico L. D., Rebeiz G. M.: 'Digital communications using selfphased arrays', IEEE Transactions on Microwave Theory and Techniques, 2001, 49, pp. 677-684

[11] Griesser T., Balanis C.: 'Backscatter analysis of dihedral corner reflectors using physical optics and the physical theory of diffraction', IEEE Transactions on Antennas and Propagation, 1987, 35, pp. 1137-1147

[12] Haddad H., Loison R., Gillard R., Jrad A., Harmouch A.: 'Compression technique for retrodirective reflectors using transformation optics', 2016 IEEE Middle East Conference on Antennas and Propagation (MECAP), 2016, pp. 1-4

[13] Lipuma D., Meric S., Gillard R.: 'RCS enhancement of flattened dihedral corner reflector using reflectarray approach', Electronics Letters, 2013, 49, pp. 152-154

[14] 'ERC Decision of 6 March 2015 on the frequency bands to be designated for the temporary introduction of $\mathrm{Au}$ tomotive Short Range Radars (ECC/DEC/(04)10) ', http://www.erodocdb.dk/Docs/doc98/official/pdf/ECCDEC0410.PDF, 2015

[15] Srour H., Gillard R.,Meric S., Seetheramdoo D.: 'Improved retrodirective flattened dihedral using incidence angle correction', 2016 Loughborough Antennas and Propagation Conference (LAPC), 2016, pp. 1-14

[16] Rajagopalan H., Xu S.,Rahmat-Samii Y.: 'On understanding the Radiation Mechanism of Reflectarray Antennas: An Insightful and Illustrative Approach', IEEE Antennas and Propagation Magazine, 2012, vol. 54, no. 5, Oct. 2012 , pp. $14-38$

[17] Carrasco E., Encinar J.,Rahmat-Samii Y.: 'Reflectarray antennas: A review', Forum for Electromagnetic Research Methods and Application Technologies (FERMAT)

[18] Diaz-Rubio A., Asadchy V.,Elsakka A., Tretyakov S.: 'From the generalized reflection law to the realization of perfect anomalous reflectors', Science Advances, 2017, vol. 3, no. 8, 11 August 2017, e1602714 